and four novel genera. Front Microbiol. 2018;9:67. https://doi.org/10.3389/fmicb.2018.00067

3. Masaki T, Ohkusu K, Hata H, Fujiwara N, Iihara H, Yamada-Noda M, et al. Mycobacterium kumamotonense sp. nov. recovered from clinical specimen and the first isolation report of Mycobacterium arupense in Japan: novel slowly growing, nonchromogenic clinical isolates related to Mycobacterium terrae complex. Microbiol Immunol. 2006;50:889-97. https:// doi.org/10.1111/j.1348-0421.2006. tb03865.x

4. Kontos F, Mavromanolakis DN, Zande MC, Gitti ZG. Isolation of Mycobacterium kumamotonense from a patient with pulmonary infection and latent tuberculosis. Indian J Med Microbiol. 2016;34:241-4. https://doi.org/10.4103/ 0255-0857.180356

5. Clinical and Laboratory Standards Institute. Susceptibility testing of mycobacteria, Nocardia spp., and other aerobic actinomycetes. 3rd edition. CLSI Standard Document M24. Annapolis Junction (MD): The Institute; 2018.

6. Hoefsloot $\mathrm{W}$, van Ingen J, Andrejak $\mathrm{C}$, Angeby $\mathrm{K}$, Bauriaud R, Bemer P, et al.; Nontuberculous Mycobacteria Network European Trials Group (NTM-NET). The geographic diversity of nontuberculous mycobacteria isolated from pulmonary samples: an NTM-NET collaborative study. Eur Respir J. 2013;42:1604-13. https:/ / doi.org/ 10.1183/09031936.00149212

7. Iemura-Kashiwagi M, Ito I, Ikeguchi R, Kadoya M, Iemura T, Yoshida S, et al. Soft tissue infection caused by Mycolicibacter kumamotonensis. J Infect Chemother. 2019;26:136-9. https://doi.org/10.1016/j.jiac.2019.06.013

8. Smith DS, Lindholm-Levy P, Huitt GA, Heifets LB, Cook JL. Mycobacterium terrae: case reports, literature review and in vitro antibiotic susceptibility testing. Clin Infect Dis. 2000;30:444-53. https:/ / doi.org/10.1086/313693

9. Tortoli E, Gitti Z, Klenk HP, Lauria S, Mannino R, Mantegani $\mathrm{P}$, et al. Survey of 150 strains belonging to the Mycobacterium terrae complex and description of Mycobacterium engbaekii sp. nov., Mycobacterium heraklionense sp. nov. and Mycobacterium longobardum sp. nov. Int J Syst Evol Microbiol. 2013;63:401-11. https://doi.org/10.1099/ ijs.0.038737-0

10. Davarpanah M, Azadi D, Shojaei H. Prevalence and molecular characterization of non-tuberculous mycobacteria in hospital soil and dust of a developing country, Iran. Microbiology. 2019;165:1306-14.

https:/ / doi.org/10.1099/mic.0.000857

Address for correspondence: Katerina Manika, Respiratory Infections Unit, Pulmonary Department, Aristotle University of Thessaloniki, G. Papanikolaou Hospital, Exohi 57010, Thessaloniki, Greece; email: ktmn05@yahoo.gr

\section{Misidentification of Burkholderia pseudomallei, China}

\author{
Bin Wu, Xinxin Tong, Haoyan He, Yinmei Yang, \\ Huling Chen, Xiao Yang, Banglao Xu
}

Author affiliation: Guangzhou First People's Hospital, South China University of Technology, Guangzhou, China

DOI: https://doi.org/10.3201/eid2703.191769

We report a case of melioidosis in China and offer a comparison of 5 commercial detection systems for Burkholderia pseudomallei. The organism was misidentified by the VITEK 2 Compact, Phoenix, VITEK mass spectrometry, and API 20NE systems but was eventually identified by the Bruker Biotyper system and 16S rRNA sequencing.

B urkholderia pseudomallei is the cause of melioidosis, a serious disease endemic to Southeast Asia and northern Australia (1). Because of the increase in international travel, the disease is now occurring in areas to which B. pseudomallei is not endemic. In these previously unaffected areas, laboratory staff might be unfamiliar with the organism or use identification systems that are not suitable for its detection, potentially leading to misidentification (2). We report the misidentification of $B$. pseudomallei by various commercial detection systems.

On May 15, 2019, a man 33 years of age in Guangxi Province, China, sought treatment for leg pain at a local hospital in Guangxi Province. Physicians diagnosed his condition as gout and prescribed oral febuxostat. However, the pain progressively worsened, and the patient began to have difficulty walking. On June 10 he was admitted to Guangzhou First People's Hospital. Laboratory analysis of serum samples taken at admission showed moderate systemic inflammation with elevated levels of procalcitonin $(0.296 \mathrm{ng} / \mathrm{mL}$; reference value $<0.05 \mathrm{ng} / \mathrm{mL})$, C-reactive protein $(61.7 \mathrm{mg} / \mathrm{L}$; reference value $<6.0 \mathrm{mg} / \mathrm{L}$ ), erythrocyte sedimentation rate $(120 \mathrm{~mm} / \mathrm{h}$; reference value $<15 \mathrm{~mm} / \mathrm{h})$, leukocytes $\left(13.87 \times 10^{9}\right.$ cells/L; reference value 1.1-3.2 $\times 10^{9}$ cells/L), and neutrophils $\left(9.42 \times 10^{9}\right.$ cells/L; reference value: $1.8-6.3 \times 10^{9}$ cells $/ \mathrm{L}$ ). His temperature fluctuated between $38.5^{\circ} \mathrm{C}$ and $39.8^{\circ} \mathrm{C}$, peaking in the evening. Magnetic resonance imaging results suggested osteomyelitis. We conducted surgical debridement and collected pus from the lesion for microbiological analysis. We used the matrix-assisted laser desorption/ionization time-of-flight (MALDI-TOF) mass spectrometry VITEK 2 Compact system (bioMérieux, 

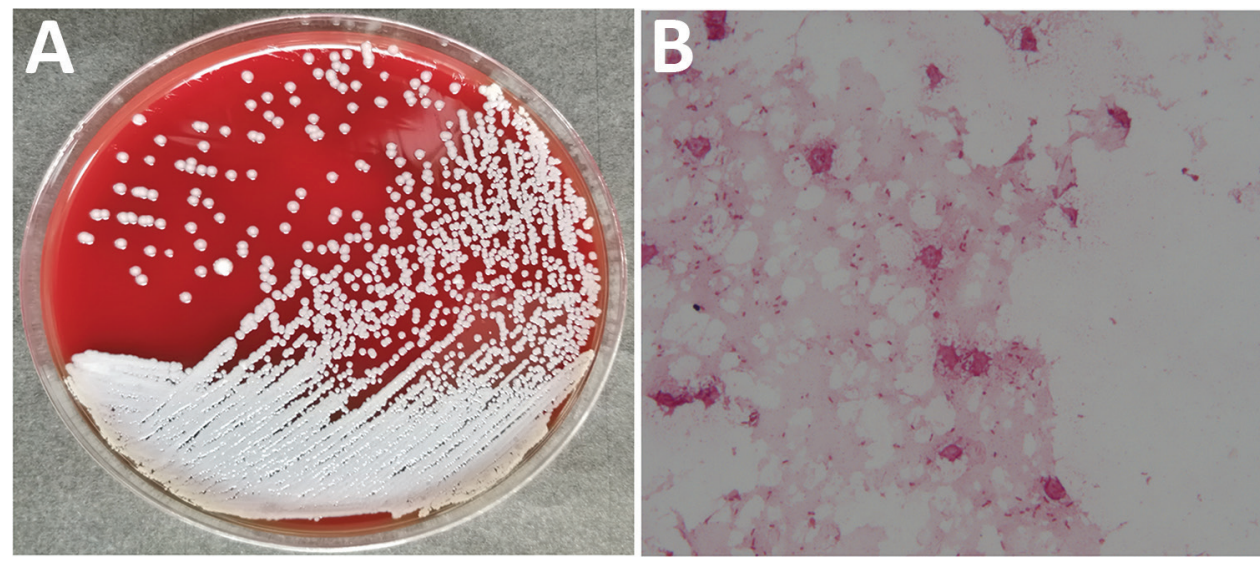

Figure. Burkholderia pseudomallei isolated from patient, China, 2019. A) Culture on sheep blood agar. B) Gram-stained smear. Original magnification $\times 1,000$ https://www.biomerieux.com) to identify the isolate as Aeromonas sobria with $93 \%$ probability. According to the VITEK 2 Compact system, the isolate was sensitive to amikacin, meropenem, imipenem, ceftazidime, ciprofloxacin, trimethoprim/sulfamethoxazole, and piperacillin/tazobactam but resistant to cefepime and aztreonam. We made a preliminary diagnosis of Aeromonas infection and treated the patient with piperacillin/tazobactam (500 $\mathrm{mg}, 4 \times / \mathrm{d}$ ) and levofloxacin $(500 \mathrm{mg} / \mathrm{d})$. However, we doubted the accuracy of this identification because Aeromonas sobria rarely causes extraintestinal disease (3). To examine this suspicion, we collected blood samples and incubated them in the Bact/ALERT 3D automated microbial detection system (bioMérieux). We cultured the samples on sheep blood and chocolate agar, revealing gramnegative rod-shaped bacteria (Figure; Appendix Figure 1, https:/ / wwwnc.cdc.gov/EID/article/27/3/191769-App1.pdf). We then tested the samples with a variety of commercial detection systems. The VITEK 2 Compact system again identified the blood sample as Aeromonas sobria with $90 \%$ probability. However, the Bruker MALDI-TOF Biotyper system (Bruker Daltonics, https:/ / www.bruker.com) identified the isolate as B. pseudomallei with an identification score of 2.18 (a score of $>2.0$ is considered an accurate identification). BD Phoenix M50 (Becton Dickinson, http://www. bd.com) identified it as Alcaligenes faecalis with $98 \%$ probability; VITEK MS (bioMérieux) identified it as $B$. thailandensis with an identification score of 2.23; API
20NE (bioMérieux) identified it as Pseudomonas fluorescens with $75.8 \%$ probability (Table).

To confirm the identity of the organism, we extracted DNA from blood cultures using a bacterial genomic DNA isolation kit (Sangon Biotech Co., Ltd, https:/ / www.sangon.com). The 16S rRNA gene was amplified and sequenced by Sangon Biotech Co., Ltd. The isolate showed 100\% identity and $100 \%$ coverage with a sequence of B. pseudomallei collected in India in 2019 (GenBank accession no. CP040552.1). On June 25, we diagnosed melioidosis in the patient. The patient recovered and was discharged after 14 days of the original piperacillin/ tazobactam and levofloxacin treatment regimen. The global recommendations from the US Public Health Emergency Medical Countermeasures Enterprise suggest that physicians treat melioidosis with intravenous ceftazidime or meropenem, according to the severity of the disease; alternatively, physicians can prescribe oral trimethoprim/sulfamethoxazole or amoxicillin/clavulanic acid (4).

We conducted multilocus sequence typing as described previously (5). This isolate belongs to sequence type (ST) 550, corresponding with isolates previously documented in Vietnam in 2005 (6). The patient in this study had never been to Vietnam, but Guangxi Province borders that country. We constructed a phylogenetic tree with 1,000 bootstrap replicates using the unweighted pair group method with arithmetic averages in MEGA X software

\begin{tabular}{lcc}
\hline Table. Identification of Burkholderia pseudomallei by various detection systems, China, 2019 & Characteristics \\
\hline Detection method & Identification result & $90 \%$ probability \\
\hline Vitek 2 Compact & Aeromonas sobria & $98 \%$ probability \\
Phoenix & Alcaligenes faecalis & 2.18 score $^{*}$ \\
Bruker Biotyper MS & Burkholderia pseudomallei & 2.23 score* \\
Vitek MS & Burkholderia thailandensis \\
API 20NE & Pseudomonas fluorescens & $75.8 \%$ probability \\
16S rRNA & Burkholderia pseudomallei & GenBank accession no.CP040552.1 \\
\hline
\end{tabular}


(https://www.megasoftware.net). This tree included isolates from other countries in Asia downloaded from PubMLST (https:/ / pubmlst.org); the isolate in this study was most closely related to ST175 from Thailand (Appendix Figure 2) (6).

The accuracy of the identifications made by VITEK $2(63 \%-81 \%)$, Phoenix $(0 \%-28 \%)$, and API 20NE (37\%-99\%) systems varied substantially $(7,8)$. Zakharova et al. found that commercially available biochemical identification systems commonly misidentified B. pseudomallei as Chromobacterium violaceum or B. cepacia complex (9). We found that although the isolate in this study was misidentified by multiple systems, most systems accurately identified the genus. MALDI-TOF mass spectrometry is a rapid, accurate, and highly reproducible technique for bacterial identification. Several studies have explored the potential of MALDI-TOF mass spectroscopy for the identification of B. pseudomallei. We prefer the Bruker Biotyper system, which is more accurate because the VITEK databases lack reference spectra for B. pseudomallei (10). In conclusion, scientists must be aware of the potential misidentification of B. pseudomallei by automated identification systems, especially those in regions to which $B$. pseudomallei is not endemic.

\section{About the Author}

Mr. Wu is a member of the Department of Laboratory Medicine of Guangzhou First People's Hospital, Guangzhou. His primary research interest is bacterial infections.

\section{References}

1. Chewapreecha C, Holden MT, Vehkala M, Valimaki N, Yang Z, Harris SR, et al. Global and regional dissemination and evolution of Burkholderia pseudomallei. Nat Microbiol. 2017;2:16263. https://doi.org/10.1038/nmicrobiol.2016.263

2. Kiratisin P, Santanirand P, Chantratita N, Kaewdaeng S. Accuracy of commercial systems for identification of Burkholderia pseudomallei versus Burkholderia cepacia. Diagn Microbiol Infect Dis. 2007;59:277-81. https:/ / doi.org/ 10.1016/j.diagmicrobio.2007.06.013

3. Kobayashi H, Seike S, Yamaguchi M, Ueda M, Takahashi E, Okamoto K, et al. Aeromonas sobria serine protease decreases epithelial barrier function in T84 cells and accelerates bacterial translocation across the T84 monolayer in vitro. PLoS One. 2019;14:e0221344. https:/ / doi.org/10.1371/ journal.pone.0221344

4. Lipsitz R, Garges S, Aurigemma R, Baccam P, Blaney DD, Cheng AC, et al. Workshop on treatment of and postexposure prophylaxis for Burkholderia pseudomallei and B. mallei Infection, 2010. Emerg Infect Dis. 2012;18:e2. https://doi.org/10.3201/eid1812.120638

5. Godoy D, Randle G, Simpson AJ, Aanensen DM, Pitt TL, Kinoshita R, et al. Multilocus sequence typing and evolutionary relationships among the causative agents of melioidosis and glanders, Burkholderia pseudomallei and
Burkholderia mallei. J Clin Microbiol. 2003;41:2068-79. https://doi.org/10.1128/JCM.41.5.2068-2079.2003

6. Kamthan A, Shaw T, Mukhopadhyay C, Kumar S. Molecular analysis of clinical Burkholderia pseudomallei isolates from southwestern coastal region of India, using multi-locus sequence typing. PLoS Neg1 Trop Dis. 2018;12:e0006915. https:/ / doi.org/10.1371/journal.pntd.0006915

7. Zong Z, Wang X, Deng Y, Zhou T. Misidentification of Burkholderia pseudomallei as Burkholderia cepacia by the VITEK 2 system. J Med Microbiol. 2012;61:1483-4. https://doi.org/ 10.1099/jmm.0.041525-0

8. Hoffmaster AR, AuCoin D, Baccam P, Baggett HC, Baird R, Bhengsri S, et al. Melioidosis diagnostic workshop, 2013. Emerg Infect Dis. 2015;21.

9. Zakharova IB, Lopasteyskaya YA, Toporkov AV, Viktorov DV. Influence of biochemical features of Burkholderia pseudomallei strains on identification reliability by Vitek 2 System. J Glob Infect Dis. 2018;10:7-10. https:/ / doi.org/10.4103/ jgid.jgid_39_17

10. Lau SK, Sridhar S, Ho CC, Chow WN, Lee KC, Lam CW, et al. Laboratory diagnosis of melioidosis: past, present and future. Exp Biol Med (Maywood). 2015;240:742-51. https:/ / doi.org/10.1177/1535370215583801

Address for correspondence: Banglao Xu, Guangzhou First People's Hospital, School of Medicine, South China University of Technology, Department of Laboratory Medicine, 1 Panfu Rd, Guangzhou, 510180, China; email: eyxubl@scut.edu.cn

\section{Autochthonous Case of Pulmonary Histoplasmosis, Switzerland}

\author{
Yvonne Schmiedel, ${ }^{1}$ Annina E. Büchi, ${ }^{1}$ \\ Sabina Berezowska, Alexander Pöllinger, \\ Konrad Mühlethaler, Manuela Funke-Chambour
}

Author affiliations: Basel University Hospital, Basel, Switzerland

(Y. Schmiedel); Hôpital du Jura, Delémont, Switzerland

(Y. Schmiedel); Inselspital, Bern University Hospital, University of Bern, Bern, Switzerland (Y. Schmiedel, A.E. Büchi, A. Pöllinger, M. Funke-Chambour); Lausanne University Hospital and University of Lausanne Lausanne, Switzerland (S. Berezowska); Institute of Pathology, University of Bern, Bern, Switzerland (S. Berezowska); Institute for Infectious Diseases, University of Bern, Bern (K. Mühlethaler)

DOI: https://doi.org/10.3201/eid2703.191831

${ }^{1}$ These authors contributed equally to this article. 